\title{
APPLICATION OF CONDUCTING ORGANIC SOLIDS FOR BIOLOGICAL SENSORS: SUCCESSES AND PROBLEMS
}

\author{
M. KRYSZEWSKI \\ Centre of Molecular and Macromolecular Studies, Polish Academy of Sciences \\ Sienkiewicza 112, 90-363 Eódź, Poland \\ Polymer Institute, Technical University, Żwirki 36, 90-924 Łódź, Poland
}

\begin{abstract}
Studies on the basic properties and fabrication of biosensors based on conducting organic polymers have been reviewed. Several types of biosensors and some classes of conducting polymers used in biosensors are described. It is shown that their selective responses towards a dissolved analy te have lead to their successful application in a new generation of ion, molecular and biosensors. Some important open problems in this fast growing field have also been indicated. They are related with the complex interactions of biomolecules and/or cells with the conducting transducers. The present tendency to extreme miniaturization of biosensors related to the utilization of a few biomolecules and/or a single cell as a heart of biosensing devices seems to be very close to molecular electronics.
\end{abstract}

PACS numbers: $81.60 . \mathrm{Jw}, 82.65 .-\mathrm{i}$

\section{Introduction}

The purpose of this contribution is to report on some applications of conducting organic solids which recently have been extensively investigated. It is easy to enumerate the list of organic conductors which have been applied in different important domains of technology and science. To this group it is necessary to include, besides organic conductors, electroluminescent and nonlinear optical systems, transistors and sensors. Conducting polymers (and in some cases low molecular weight solids) are perfect materials with which one can produce sensory devices since their chemical composition and electronic perturbations may vary by more than a factor of $10^{13}$ [1].

Many previous investigations have been devoted to conducting polymer-based gas sensors, however, most of these systems are chemically irreversible and have a time dependent (i.e. integrated) response $[2,3]$. The main achievements recently have been in the biosensor areas. They draw particular attention because 
they are very close to molecular electronics - the main effects are occurring on a molecular basis.

In this report we summarize the different approaches to conducting polymer based biosensors. In principle however the design of a sensor can be applied to any chemical entity since the molecular nature of the sensory materials allows the tailoring of polymer's response to a given chemical species by the implantation of known molecular recognition and/or chemical sensitivity.

\section{General remarks}

A biosensor is an analytical device in a functional contact with a transducer to monitor the local environment. The essential features of such devices are that the biological material produces a quantitative signal in response to a specific analyte. The signal can be measured by the transducer which may be an electrode, by an optical detector, a piezoelectric crystal or any variety of other detector systems. Biosensors have been designed to fulfil a number of criteria which include good reliability, simplicity of use (preferably reagentless), high selectivity and sensitivity in response to analyte [4].

Biosensors are frequently defined in various ways. We shall adopt a well-utilised definition: a biosensor comprises an analyte selective interface in a close vicinity or integrated with a transducer whose function is to relay the interaction between the surface either directly or through a chemical mediator (Fig. 1).

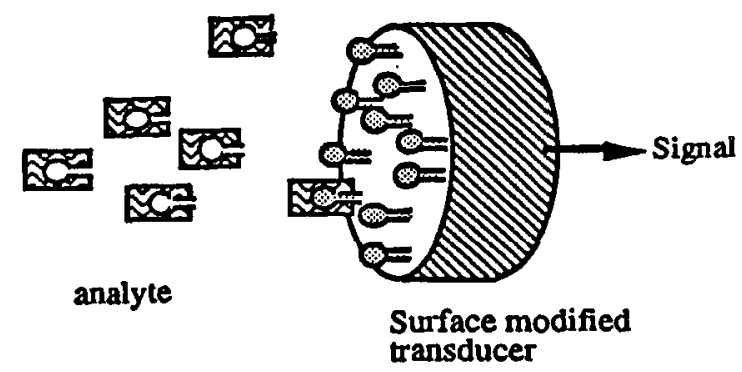

Fig. 1. The biosensor concept.

The type of transducer and the transduced parameters will depend on the kind of biological event concerned with a detection of the analyte. One can say, e.g. that the system designed to immunoassay when an antibody-antigen interaction must be detected by an appropriate enzyme linked redox reaction. In some cases several parameters can often alter during an analytical pathway, the choice of device is many times connected with many transducers. The multiple nature of the parameters which have been taken into consideration are schematically presented in Fig. 2.

Depending on reaction being of interest one should select a right transduction parameter. Many methods are known to detect the signal or changes of the system due to the reaction at the biosensor surface. Electrical response can be 


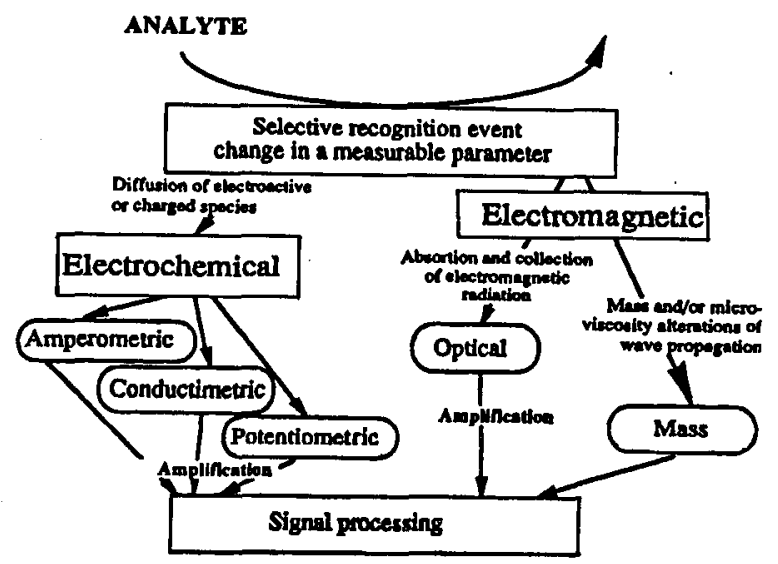

Fig. 2. Transduction parameters and various devices types redrawn after [3].

detected in three basic ways: amperometric, potentiometric and by conductivity measurements. These three methods will be the main object of our discussion because in many of them conducting organic solids have been used. It seems however necessary to mention briefly optical sensors and other types of sensors based on complex processes of analyte and the solid surface.

Optical biosensors are working in two modes depending on the optical configuration. The intrinsic mode uses the incident wave not directed through the bulk sample, but propagating along a wave guide and interacting with the sample surface within the evanescent field. The extrinsic mode uses the light passing through the sample phase and interacting with the sample.

In the traditional biosensors optical measurements are made in a solution without immobilized reagents. The most sensitive optical sensors of this type are based on fluorescence measurements. Fluorescent labels are often used for probing binding events (e.g. antibody-antigen complex formation). A typical sensor might use fibres to guide the light to and from the end of the fibre, where the sensing chemistry is localized. Fluorescent indicators are often chosen to have long wavelength absorption and emission maxima. The most widely employed dyes for immunoassay have been fluorescein derivatives and rhodamine derivatives. Time resolved immunoassays are also made. Here we shall not describe several possible sensor configurations but it is worthwhile to mention fluorescence polarization measurements which can offer the possibility of a multidimensional analysis which makes easier to draw conclusions about molecular weight changes of antibody or/and about immobilization of reagents.

In the intrinsic mode which is equivalent to the extrinsic optical sensor it is the wave guide where the reagents are immobilized on the surface within the evanescent field. In this configuration the excitation light does not pass through the bulk of the sample and the interaction between the surface immobilized reagents is smaller. In the ray model for propagation of light through a guide the interaction with the surface occurs at the points of total internal reflection within the 
wave guide. A thin film wave guide increases the interaction but it is difficult to construct.

In the literature several other optical methods have been described to give signal enhancement in biosensors. Some of them are based on modulation of the field excited at the interface between different materials due to incident light. The surface plasmon resonance is the most important. Current developments in this area investigate refractive index labels and other optical labels, too.

It seems necessary to mention also the use of acoustic waves and microbalance to measure the changes of the attached antigen. To these methods the piezoelectric detection belongs too. The detailed discussion of these techniques is beyond our discussion which is mainly devoted to the electrical sensors based on conducting organic solids.

The immobilization matrix may function purely as a support, it may be conccrned with the mediator of the signal transduction mechanism associated with the analyte. Immobilization techniques are usually divided into the following groups:

1. Physical entrapment in an inert matrix (membrane);

2. Physical adsorption;

3. Chemical adsorption;

4. Physical retention in a polymer matrix;

5. Binding to a functionalized support;

6. Entrapment in an "active" membrane.

These factors will be discussed for one simple amperometric biosensor used for the detection of glucose. The specific influence of the immobilization process of the recognition molecules is important for all types of biosensors and their reactivity with the analyte.

Entrapment in an active membrane is the method of particular interest. The membrane may trap the enzyme and enhance the generated charge transport that is why conducting polymers are often used in the construction of biosensors.

Physical entrapment in an inert polymer matrix of enzymes or of whole cells is usually achieved by the cross-linking of the gel. It is also possible to use low temperature cured sol-gel silicon glasses. This method is a very slow one. For this reason it is not the ideal one for less stable biological molecules. Nevertheless "bioactive glasses" are an interesting concept. Usually polymers like poly(vinylalcohol), poly(vinylpyridine), gelatine etc. are used. The best seems to be dialdehyde cross-linked poly(acrylamide). There are some problems connected with the practical application of such matrices. They are mainly related with the stabilization of the biorecognition molecule.

Physical adsorption of the biorecognition molecule is the simplest immobilization process. In that case one should take into consideration the orientation or site attachment (the active site of the enzyme must be free to make the effective antibody-antigen complex formation).

Chemical adsorption, it is the chemical attachment, is mostly explored for protein immobilization. Coupling frequently involves surface $\mathrm{NH}_{2}$ groups on the 
proteins and results in a very varied surface coverage density. This method is effective for large surfaces (microspheres) and less useful for planar transducers. Physical retention in a polymer matrix is similar to physical entrapment with all advantages and disadvantages.

Binding to functionalized support is similar to chemisorption. Binding of an enzyme is connected with an adequate treatment of the matrix which results in a formation of desirable active groups being involved in direct binding of e.g. protein.

At first the mostly used amperometric detection will be discussed.

\section{Amperometric biosensors}

The first described biosensor used "enzyme" electrode applied glucose oxidase as a selective biorecognition molecule for glucose. The enzyme was next to platinum electrode in a membrane sandwich. The $\mathrm{Pt}$ electrode, polarized at t0.6 V vs. SCE respond to hydrogen peroxide produced

Glucose $+\mathrm{O}_{2} \stackrel{\text { glucose oxidase }}{\longrightarrow}$ Gluconic acid $+\mathrm{H}_{2} \mathrm{O}_{2}$.

The amperometric systems utilising redox enzymes are very popular. However, as follows from the scheme presented above, the electrode transfer between enzyme and electrode is not simple because the monitored signal was the product of transformation - hydrogen peroxide. The transduced signal is indirect one.

In practical terms, as far the biosensor is concerned, this may be of no consequence. However utilizing $\mathrm{H}_{2} \mathrm{O}_{2}$ as a measured species requires a constant supply of oxygen and a measuring potential. In order to increase the performance and repeated efficiency the synthetic replacement was found for oxygen. One can modify electrodes and enzyme by mediators. Many electron acceptor molecules and complexes have been considered for a role of mediator. Amongst the favourites ferrocene, ( $\eta^{5}$-bis cyclopentadienyl-iron) and its derivatives still attract attention $[5,6]$.

In this cyclic reaction the only molecule which is now consumed by the sensor is the analyte itself and the other reagents are regenerated. Such mediator-linked assays, even at the simplest, one enzyme level, can be an elegant solution for a biosensor. It must be mentioned that the reagent must be immobilized to interact with both analyte and transducer as a self-contained system before the "biosensor" label is attached.

In the small amperometric biosensor field the reagent may by different in nature. In particular, the very nucleus of the analyte selective reaction is not always an isolated enzyme, but may be a whole cell or cellular component. Microbial biosensors with a specificity for analytes ranging from $\mathrm{CO}_{2}$ to Vitamin $\mathrm{B}$ are known. In majority cell based devices relate on the change of $\mathrm{O}_{2}$ tension monitored at an electrode mounted behind the cell layer. Like the isolated enzyme counterparts artificial redox mediators have been investigated but in this case transport is restricted by the cell walls and membranes. It was shown that a considerable variation in efficiency of mediator occurs, even between similar mediators. Different factors (e.g. lipophilicity) and surface interactions play sometimes a more important role than an enzyme preparation. 


\subsection{Amperometric biosensors based on poly(pyrrole)}

There is an important problem which needs to be discussed. It is known that conducting poly(pyrrole) (PPy) is used in biological sensors. It has positive charges delocalized along the backbone and counter ions $\mathrm{X}^{-}$(dopants). It is very stable in aqueous media and electrochemically reversible. That is why it is used in various biomedical applications like drug delivery [7] or glucose sensing films [8]. In many situations the key aspect is the biocompatibility. It will be addressed in the following. It is of importance to evaluate this material towards protein absorption, cytotoxicity and both structural and electrochemical stability in physiological environments. Protein binding properties of PPy pellets [9] and PPy + PPS - films [10] shall be discussed.

The problem consists whether the cell can grow on PPy substrates and how it depends on various dopant anions [11]. Several dopants starting from $\mathrm{Cl}^{-}$up to polyanions (e.g. poly(styrenenesulfonate) $\left(\mathrm{PPS}^{-}\right)$) and various cell lines namely CPAE (cow pulmonary artery endothelium), NRK (normal rat kidney epithelium) and VA-13 (transformed fibroblasts) were investigated. Poly(pyrrole) thin films were electrochemically grown on gold electrodes and sodium salts of dopant anion as the electrolyte, at constant potential of $0.7 \mathrm{~V}$ versus $\mathrm{Ag} / \mathrm{AgCl}$ as a reference electrode. All cell culture was carried out at least in duplicate with all cell lines. In order to characterize the protein absorption contact angle measurements were made for the films before (bare films) and after the protein adsorption. The obtained PPy films with absorbed proteins, were soaked in the culture medium for 3-4 minutes and then washed carefully with water to remove any loosely absorbed proteins and finally dried*.

For the evaluation of cell growth and proliferation on the $\mathrm{PPy}^{+} \mathrm{X}^{-}$film surfaces it is necessary to have a system with which one can compare cell growth characteristics. Gold makes possible such a comparison because it can sustain cell growth and proliferation without indicating any cytotoxicity. The cell proliferation was observed within 18-24 h. The cells reached confluence and continued to live even after a period of 2 weeks.

First studies concerned the role of big anions like PPS - . The proliferation of V-13 cell line was on PPy+ PPS- films as dense as on a gold surface and they did not exhibit any cytotoxicity even after a period of 2 weeks. Similar results were obtained with other cell lines.

Analogous experiments were carried out on PPy films with small dopant ions like $\mathrm{Cl}^{-}$or tosylate $\mathrm{CH}_{3}-\left(\mathrm{C}_{6} \mathrm{H}_{4}\right)-\mathrm{SO}_{3}\left(\mathrm{TsO}^{-}\right)$using VA-13 cell line (this is extremely sensitive towards protein coated substrates). It was found that $\mathrm{PPy}^{+} \mathrm{Cl}^{-}$ films were a little inferior than $\mathrm{PPy}^{+} \mathrm{PPS}^{-}$. On the other hand, experiments on films with a more hydrophobic anion like tosylate $\mathrm{TSO}^{-}$have shown that the cells had a greater difficulty to attach to the surface and even after $96 \mathrm{~h}$ only some cells were spread on the surface. Using more hydrophobic counterions, e.g. p-dodecylbenzensulfonate (DBzS) or dodecylsulphate (DS) with the same cell cul-

*In this paper we have omitted the detailed description of the investigated cells and cell culture medium. These preparations correspond to the standarts in biochemical laboratories. Some remarks concern the characterization of cell cultures. 
ture one observes a similar cell adhesion like in the case of PPy+TSO-- Thus it is possible to infer that chemical nature of the dopant has a significant influence on the type of protein that is preferentially adsorbed from solutions. It can be speculated that these differences are coupled with surface conformational changes of the absorbed protein (attachment, spreading and proliferation). This has been confirmed by changes of advancing contact measurements of $\mathrm{PPy}^{+} \mathrm{X}^{-}$thin film before and after protein absorption (e.g. after protein absorption for $\mathrm{PPy}^{+} \mathrm{PPS}^{-}$ it was $38^{\circ}$, whereas for $\mathrm{PPy}^{+}$Tosylate ${ }^{-}$it was $68^{\circ}$ ).

In conclusion it was shown that PPy thin films are not toxic and the surface properties of these films towards the cell attachment, spreading and growth can be modified by the selection of an appropriate dopant ion.

In the above-mentioned studies PPy was obtained electrochemically on gold in the presence of various counterions. There is still a question whether the substrate plays a certain role in the protein adsorption or cell attachment. An electrochemical synthesis of PPy was also carried out on ITO (indium tin oxide) electrode with tetraethylammonium- $p$-toluene sulphonate in a classical way. The PPy films were characterized by conductivity measurements and spectrophotometry [12] as well as by static wetting contact angles investigations.

Fibronecetine (FN) was mixed with ${ }^{125}$ I labelled FN and used as a model protein. Its absorption was quantitated according to a previously described method [13] in conditions which maximize the adsorption efficiency. Cell attachment of Balb/c $3 \mathrm{~T} 3$ mouse fibroblasts was performed both in the presence and absence of calf serum.

The PPy surfaces were sterilized via exposure to UV irradiation for $30 \mathrm{~min}$. The unattached cells were removed with phosphate buffer saline (PBS). The attached cells were then removed by trypsinization and were counted (attachment was determined by the counts of the trypsinized cells divided by the total counts from the wash and the trypsinized cells). Cells after an appropriate fixation were observed under a phase-contrast microscope. PPy films were also prepared by the known chemical oxidative method. The thickness of such films equal to about $700 \AA$ was estimated from absorbance in their oxidized state. The conductivity measurements confirmed the oxidized state of the PPy both deposited at tissue culture (TC) or bacteriological grade (PD) polystyrene. They have been uniform as determined from wetting angle measurements $\left(77 \pm 1^{\circ}\right)$. In this study it was also demonstrated that thin films of oxidized PPy adsorb the proteins and that the cells exhibit a normal response such as attachment or spreading when cultured on PPy. It seems that these are not cell specific receptors for PPy but there is probably an electrostatic interaction between the cell adhesion proteins and PPy.

At the end of the discussion on the protein and cell attachment it should be concluded that this conducting polymer may support cell functions thus being suitable as the basis of sensors for cell culture studies. This very positive result is only partially limited by the consideration on the interfacial effects.

Proteins are surface active molecules having a natural tendency to be present at phase boundaries as a result of their amphiphilic and macromolecular nature. Protein absorption will occur at any interface in contact with a protein-containing fluid. It has important consequences (e.g. protein absorption is considered to be 
an initiating event in the body's response to implants (biocompatibility) as it was shown for biosensors. Current views comprise the adsorption process including kinetics, thermodynamics and protein-surface binding modes.

Proteins are generally large molecules and they can contact a surface at more than one point. So they are chemically heterogeneous with the surface region that are hydrophobic, polar and charged either negatively or positively. The solid absorbing surface will normally have a similar range of sites, so a number of binding mechanisms are possible. The relatively well-defined normal size and shape of protcins result in different orientation even when the dimensions are retained. It is also possible that the shape will be changed via conformational alternation under the influence of the surface. These effects have an influence on the adsorption process and slow desorption although an exchange between the adsorbed and dissolved proteins have been observed. The lack of demonstrable reversibility leads to a discussion regarding the application of thermodynamical analysis to absorption data in spite of many works devoted to such an analysis. It seems worthwhile to note that the response of the adsorbed layers to electrolytes demonstrate electrostatic interactions.

Orientation can in principle be studied by the use of antibody binding using antibodies to local epitopes on the protein. However it is difficult to distinguish between orientation effects and conformational changes using this method. In multiprotein systems there is generally a distribution between the solution and the interface which reflects the concentrations, diffusion coefficients and surface binding affinities of the component proteins. The present level of understanding is not sufficient but it can be concluded that the initial layer composition is determined by transport rates whereas the ultimate composition is determined by binding affinities. It appears that there is a sequence whereby proteins of relatively low affinity and low concentration are displaced by those of relatively high affinity and low concentration.

It must be noted that the denaturation is not a concomitant factor of adsorption. Many proteins have been shown to retain biological function in the adsorbed state including enzymes and antibodies. Adsorbed antibodies state including enzymes and antibodies and antigens form, as we saw before, a basis for solid state immunoassays. There are however many examples where demonstrable protein alternation does occur on adsorption. Physical alternation was observed by a spectroscopic method: e.g IR frequency changes of bound proteins which indicate how strong their attachment is, as well as circular dichroism studies which show a loss of alpha helical content in adsorbed proteins.

Protein adsorption influences the cell adhesion which is mediated by them. Some proteins, e.g. fibrinogen, promote cell adhesion whereas plasma albumin has an inhibitory effect. Recent studies on the molecular mechanism of cell adhesion suggest that ligands in adhesive proteins (adhesins) consisting of a few aminoacids interact with specific receptors in the cell membrane.

From the above discussion it is clear that a control over protein adsorption could be advantageous in many applications, e.g. to control the composition of adsorbed layers for a given objective. Another example is the minimization of the cell adhesion. 
From the above discussion it follows that the complex process of protein adsorption is important, particularly for the full understanding of biosensors activity and the obtained results. It leads sometimes to discrepancies in the values determined in different laboratories for the same substance.

There are other applications of PPy in biological sciences. PPy and poly(sodium 3-pyrrole butene-sulfonate) $\left(\mathrm{PPy}^{+} \mathrm{BS}^{-}\right)$interacts with soluble collagene and porcine pericardium. In this way new hybrids characteristic of conductivity which depends on collagen content were obtained. The $\mathrm{PPy}^{+} \mathrm{BS}^{-}$is more active thus the higher is collagen content in this hybrid the higher is its conductivity. Polymerization of Py or PyBS in porcine pericardium results in black thin films [14].

Cytotoxicity of these materials has not been fully known as yet. One expects anticalcification potential of the hybrid tissue. This observation is important for further medical studies.

Polyaniline (Pani) seems to be not an inert matrix when glucose oxidase (GOD) is entrapped in its structure. The presence of glucose in the modified electrode, polarized at $+0.6 \mathrm{~V}$ vs. SCE results in a current increase with time (Fig. 3). The $\mathrm{d} i / \mathrm{d} t$ is proportional to glucose concentration (some direct or indirect interaction with the polymer!). It is not connected with $\mathrm{H}_{2} \mathrm{O}$ since it is independent of $\mathrm{O}$ concentration. The mechanism of Pani activity has not been fully understood thus far but it shows that conducting polymers can be useful matrices which interact with the redox enzymes (Fig. 3).

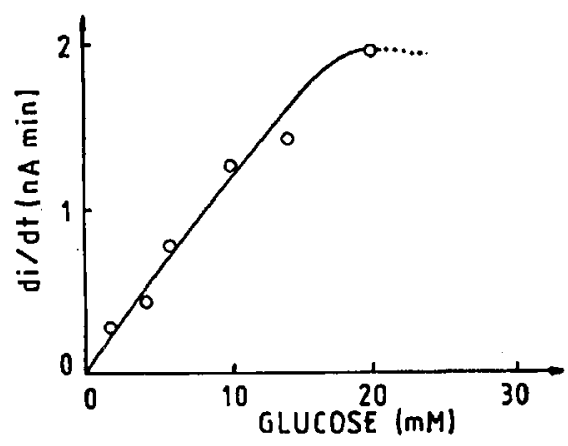

Fig. 3. Dependence of $\mathrm{d} i / \mathrm{d} t$ response on glucose concentration at $+0.65 \mathrm{vs}$. SCE at glucose oxidase/Pani electrode.

There is a tendency to make a comparison of biosensors with based on conducting redox polymers. Some words of caution belong to the redox polymers, e.g. poly(vinylferrocene) in which the redox centre is an integral part of the macromolecule. It seems to be the best, but the heterogeneous charge transfer rate constant is typically $10^{-2}-10^{-3}$ lower than the solution species as it was found a long time ago [15]. Redox polymers based on polysiloxanes containing ferrocene in the side chains, mixed with carbon are used for making electrodes. Their efficiency depends on the length of side chains and content of ferrocene. It is not 
sure if the total absence of $\mathrm{O}_{2}$ is possible. The mediate signal is smaller at high glucose concentration. Summarizing, redox polymers show interesting properties (mediator immobilization by electrostatic forces at the surface). Although charge transfer kinetics is proficient but some leaching of mediators out of polymers is observed.

All open questions mentioned before for biosensors with conducting polymers are valid for redox polymers as well, and in many cases they are probably even more important.

\section{Potentiometric biosensors}

Potentiometric biosensors are usually used for an analyte which cannot be assayed via redox enzyme (electron transfer). Potentiometric measurements have been mostly developed around $\mathrm{pH}$ sensitive electrodes. Any of the enzyme pathways which result in a change of $\mathrm{pH}$ concentration can be applied here. In most cases penicilinase or urease was used

$$
\begin{aligned}
& \text { Peniciline } \stackrel{\text { penicilinase }}{\longrightarrow} \text { Penicilloate }+\mathrm{H}^{+}, \\
& \text {Urea } \stackrel{\text { urease }}{\longrightarrow} \mathrm{NH}_{4}^{+}+\mathrm{HCO}^{-3} \text {. }
\end{aligned}
$$

The final response to the $\mathrm{pH}$ changes depends on the balance of all equilibria involving $\mathrm{H}$ protonation and deprotonation reactions of the enzyme reaction products. The theoretical kinetic model, considering all these associated transport phenomena, predicts the steady state response of an enzyme based $\mathrm{pH}$ sensor [16].

Novel application of potentiometric biosensor and of field effect transistor (FET) devices involves antibody-antigen formation [17]. The application of various FETs as biosensors is a large field of activity in the immunoassays. A discussion of the proposed devices and of mechanisms of their action cannot be included in this discussion, although many laboratories are active in this evidently interesting field of biosensors studies.

\section{Ion selective sensors}

Systems containing ionophores which are ion selective have selective response. Typical polymeric material is PVC with macrocyclic receptors dissolved in a plasticizer. It retains only a limited amount of plasticizer and ionophore and it has a limited stability but it was adopted for immunoassay systems already some years ago [18]. Antigen digoxin coupled with potassium was immobilized in PVC membrane, too.

A molecule based sensor has to give a measurable response to various stimuli. The measurable response includes also chemically induced resistance changes in conducting polymers. Conformational and electrostatic perturbations can cause conductivity changes as high as $10^{5}$ or even greater thus conducting polymers can be very sensitive sensory materials.

At present many efforts are made to obtain ion sensitive and selective conducting polymers. They seem to be very good materials to produce sensory devices, based on the above-mentioned properties, particularly to detect alkali ions and electron deficient molecules. Such sensors are also important for ecology. 
The modern approach is to create conductivity changes which will be brought about by a novel mechanism involving the formation of barriers to carrier transport or by introducing carriers via host-guest induced doping.

The general aim is to obtain systems in which switching process is chemically reversible allowing for the formation of sensors which give a steady state response, being independent on exposure time and permitting the sensor to return to its original state once the chemical signal is absent.

\subsection{Biosensors based on poly(thiophene)}

Attention is focused on poly(thiophenes). Its high environmental stability and versatile chemistry allow for a facile synthesis of functionalized monomers. Poly(thiophenes) are only highly conductive when charge carriers are injected via redox process. The dominant carriers are positively charged bipolarons (dications which delocalize over approximately 3 rings). A bipolaron structure produces a bond alternation which requires that the centre has "quinoid" character and produces a double bond between thiophene rings (for recent review on poly(thiophenes) see [19]).

Considering this fact one can expect that the chemically induced twisting would modify the intra chain transport. Sterically bulky substituents can dramatically reduce the conductivity of poly(thiophenes).

The established nature of crown ethers chemistry makes possible to obtain ion selective twisting [20]. Crown ethers containing polythiophenes have been shown to exhibit an inochromatic activity. In these systems changes of electronic properties are due to the combination of both conformational and electronic effects (e.q. monothiophene units with oxygen atoms of polyether are attached at the thiophene $\beta$-position).

New sensors are based on conductivity changes related exclusively to electronic influences. Two new routes are described leading to interesting sensors.

\subsubsection{Sensors based on barriers to conduction}

Ion sensitive polymer I contains alkoxy substituted thiophenes and "crowned" thiophenes. Complexation of a metal ion causes an increase in oxidation potential sites along the polymer back bone resulting in a barrier which impedes carrier mobility (see scheme below).

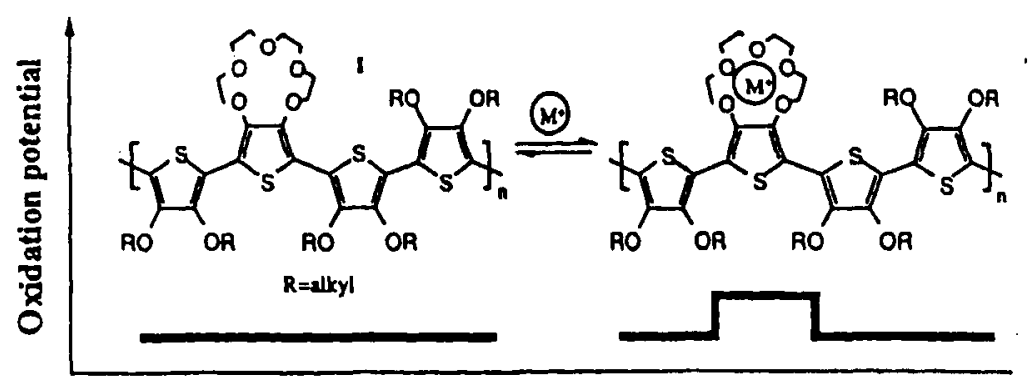




\subsubsection{Sensors based on bipolaron trapping}

Polythiophenes with thio-ethers in the $\beta$-positions exhibit a conductivity $10^{5}-10^{7}$ times lower than polythiophene. This is attributed to bipolarons trapping (i.e. localization). Similarly the oxidized states of microcycle containing polymer II should exhibit ion sensitive conductivity change (see scheme below).
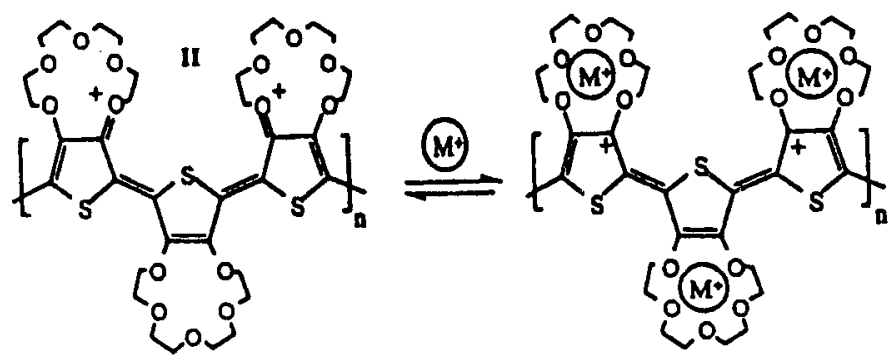

In this case the oxygen atoms of crown ethers will donate electron density towards the backbone of the polymer, trapping the carriers outside of the polymer backbone and decreasing the conductivity.

Upon the binding of an ion by macrocycle the donating ability of oxygen atoms will be reduced due to coordination of the lone pair to the ion. This effect will free the carriers leading to the increase in conductivity.

New interesting sensory materials are based on the ion selective twisting. Polythiophenes in which crown ethers linkages span the bithiophenes groups are of peculiar interest. In the uncomplexed state the polymer backbone can adopt preferred planar conformation which maximizes the conjugation. Complexation of an ion of a correct size can then induce the desired twist in the polymer backbone and change the conjugation, which in turn leads to the change in conductivity (see scheme below).

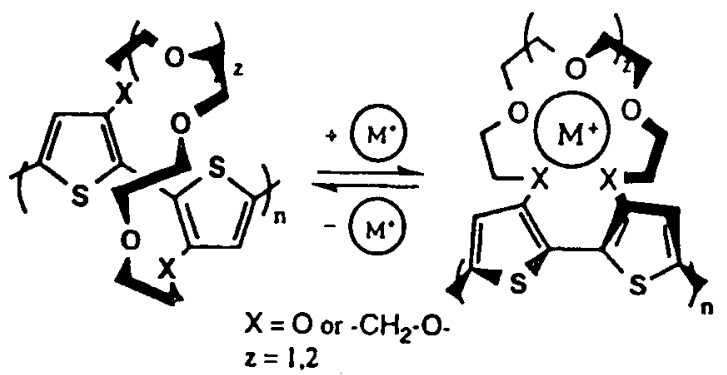

IIomo- and copolymers have been also synthesized. When $\mathrm{X}=\mathrm{O}$ (oxygen) there is a large ionochromic change (specific); consistent with twisting. For $Z=1$ the largest colour change in the presence of $\mathrm{Na}^{+}$occurs and polymers with $Z=2$ are sensitive to $\mathrm{K}^{+}$ions. Polymers with $\mathrm{X}=\mathrm{CH}_{2}-\mathrm{O}$ show a small response to ions. 
The explanation of the mechanism of conductivity change is clearly seen in thiophene monomers which have crown ethers in the 3 and 4 positions. The alkoxides donate electron density into the backbone and resonance structures which give positive charges on the alkoxide oxygen are of importance. The ion complexation will reduce the donating ability of the oxygen to poly(thiophene) and rise the oxidation potential of a particular ring. The bipolaron transport is impeded in complexed, "crowned" thiophene (see scheme below).
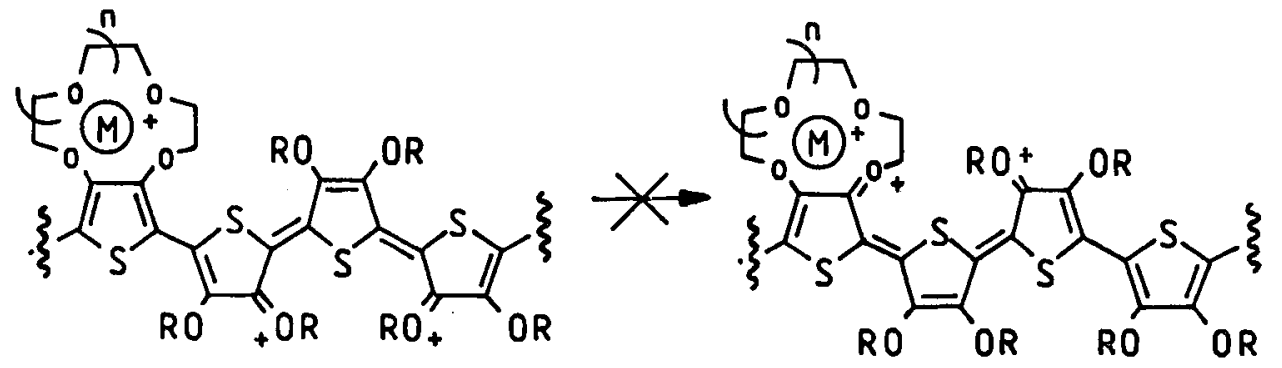

\subsection{Selective sensors for organic chemicals based on polythiophene}

IIost-guest interactions of organic molecules can be also used to produce conductive polymer based sensors for organic species. The electron rich macrocycle from rotaxane complexes with electron deficient molecules [21].

The electron-rich macrocycles - rotaxane type - containing bithiophene group have been synthesized and polymerized to produce new conducting polymers (see 1 in scheme below). They are strongly complexing electron poor molecules (see 2 in scheme below) (rotaxane complexes). It can be anticipated that these new polymers may be easily doped with strong $\pi$ acceptors and a new class of conducting polymers can be prepared.

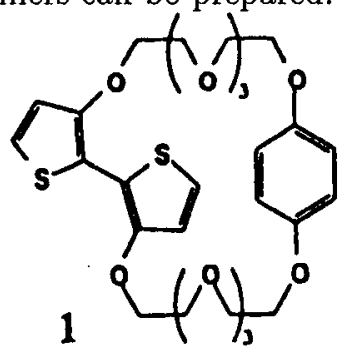

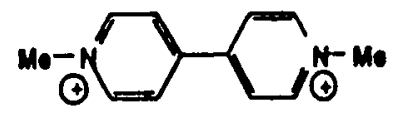

2

\section{Other systems containing conducting polymers}

It was mentioned before that the sensitivity of biosensors depends on the surface at which proteins can be immobilized, thus latexes are important elements of various diagnostic systems [22]. The suitable monodisperse latex particles with an ability to adsorb and/or covalently immobilize proteins have been prepared on the basis of styrene and acrylic monomers [23]. It is possible to observe the suspensions and/or precipitates on solid surfaces.

Polymerization of polypyrrole (PPy) lead to a new material potentially very useful for the diagnostic application in the above-mentioned sense. Doped PPy 
is deeply black, thus particles of PPy are good candidates for solid supports for diagnostic (dry diagnostic test). Redox polymerization of PPy in carefully chosen conditions and additives leads to latexes with particle diameter ranging from ca. 50-300 nm. A very effective attachment of proteins on PPy surface is insufficient in spite of charges. Derivatization techniques are known which lead to covalent immobilization of proteins, e.g. par formation of polyacrolein shell [24].

Attachment measurements of human serum albumine (HSA) and of human gamma globuline $(\mathrm{gG})$ on pure PPy and functionalized latex show that these PPy core/polyacrolein latexes are very efficient and the number of immobilized macromolecules per 1 particle can be varied in a controlled way.

It is interesting to note that two dimensional latex assemblies immobilized covalently on the surface (glass, metal, polymers) would be very convenient for fabrication of biosensors.

The preparation of such a system depends on an ability to immobilize the particles on a specific support in a two-dimensional ordered assembly. It was shown that poly(styrene)acrolein latex with aldehyde groups on the surface can be immobilized on modified quartz surface forming two-dimensional assemblies with free aldehyde groups suitable for subsequent reaction with proteins (SEM, light scattering and computer simulation) [25]. This example shows that conducting polymers (usually dark materials) may be used also in the field of latex based biosensors. However, the basic function of conducting polymers, i.e. high conductivity, has a role in the control of surface charge being an important factor for immobilization of the latex at the solid state surface and proteins on the latex.

\section{Concluding remarks}

1. The natural driving force for the development for biosensors came from a diagnostic field. Nevertheless the increasing awareness of the state of our environment and the control and monitoring air and ground species give a new incentive for biosensor science and technology.

The development of sensor devices required a separate and a linked investigation at various levels. Our basic understanding of immunoassay, enzyme linked assay, recognition proteins catalytic active sites and particularly their electronic transduction continue to occupy the field. I have shown that there are still many open problems and questions.

2. It is not simple to summarize the areas which should be particularly needed for further investigations. We have to be concerned with matching of natural and synthetic materials and technologies to enhance free communication between analyte and data handling circuits.

3. Since first stages "the Biosensor Science" have become multidisciplinary. The biosensor concept is simple but its realization is far from being simple. The integration of naturally occurring molecules with synthetic elements is not straightforward and while the nature of the possible parameters for a given analyte can be often easily identified, the mechanism of transduction is not always fully understood. 
4. After more than two decades of research we have to make fundamental investigation to understand the systems and all complex reactions and chemical modifications on the active substrate and analyte at the contact to find a real new progress in the future.

I hope that the "organic conducting material people" will play an important role in this future development but it seems necessary to say that the simple "physical" approach is not sufficient, because of the complex nature of interactions of analyte and conducting organic solid transducers.

In this brief survey progress and problems of macroscopic and quasi macroscopic molecular biosensors were discussed. In many of the above discussed biosensors the complex biological molecules were shown to be the basic part of this type of sensing devices. Nature provides excellent examples of utilization of molecules and ions as well as electrons and holes in energy and information transport. Molecular materials have already found application in electronics. In such refinement of properties the achievement of improved performance in particular end uses presents a challenge to synthetic chemists. The purification to the level essential in most applications is far from trivial. Unusual properties unique to molecular materials are discovered (both natural substances and synthetic compounds) and will be of continuing interest in utilization.

On the other hand the miniaturization of individual biosensors is important for a variety of reasons, e.g. the potential use in neurological studies, biocompatibility measurements in very small volumes, determination of spacial concentration profiles, biochips, etc. Here the development of nanotechnology and atomic resolution microscopy and its use to excite and modify molecules provide a tool without which the progress in that field would be very difficult.

Advances in the understanding of the effectiveness of miniaturized biosensors based on properties of organic solids and particularly on conductivity changes of polymers seems to be on the direct line towards molecular electronics. Now we are still dealing with a number of sensor molecules, e.g. enzymes antibodies but in some cases single cells (cellular biosensor) are used. One can expect that the progress in physics, chemistry, biology and engineering is now providing the means to conduct experiments at molecular scale. Only time will tell us what the impact on molecular electronics will come from microbiosensors science and application. Evidently research in molecular scale electronics using other routes is very challenging and necessary.

\section{Acknowledgment}

The author would like to thank Dr hab. J.K. Jeszka and Dr hab. St. Słomkowski, Center of Molecular and Macromolecular Studies, Polish Academy of Sciences, for discussions and particularly J.K.J. for redactional assistance. 


\section{References}

[1] Handbook of Conducting Polymers, Ed. T.A. Skotheim, Marcel Dekker, New York 1986.

[2] Electronic Properties of Conjugated Polymers III: Basic Models and Applications, Eds. H. Kuzmany, M. Mehring, S. Roth, Springer Verlag, Berlin 1989.

[3] Electroresponsive Molecular and Polymeric Systems, Ed. T.A. Skotheim, Marcel Dekker, New York 1987.

[4] E.H. Hall, in: Biosensors and Chemical Sensors, Eds. P.G. Edelman, J. Wang, ACS Symposium series 487, ACS Washingon 1992, Ch. 1.

[5] U. Löffler, H.D. Weinhöfer, W. Göpel, Biosensors and Bioelectronics 6, 343 (1991).

[6] J. Wang, N. Nasser, Anal. Chim. Acta 242, 259 (1991).

[7] Q-X. Zhou, L.L. Miller, J.R. Valentine, J. Electroanal. Chem. 261, 147 (1989).

[8] M.F. Mendes Viegas, E.M. Genies, M. Fouletier, E. Vieil, Electrochemica Acta 37, 513 (1992).

[9] A.B. Smith, C.J. Knowles, Biotechn. Appl. Biochem. 12, 661 (1992).

[10] L.A. Prezyna, Y.J. Qui, J.R. Reynolds, G.E. Wnek, Macromolecules 24, 4283 (1991).

[11] Venkatram R. Shastri, G.E. Wnek, Polym. Prep. 34, (2) 70 (1993).

[12] J. Wang, D. Ingber, R. Langer, ACS Polym. Prepr. 34, (2) 60 (1993).

[13] D. Ingber, Proc. Nat. Acad. Sci. USA 87, 3579 (1990).

[14] Hwee Cheze Li, E. Khor, E. Wee, IUPAC Macro-Acron 94, Abstracts, p. 697.

[15] J. Leeddy, A.J. Bard, J. Electronal. Chem. 189, 203 (1985).

[16] S. Galb, R. Koncki, A. Hulanicki, Electroanalysis 3, 361 (1991).

[17] R.B.M. Schasfoort, P. Bergveld, J. Bomer, R.P.H. Kooyman, J. Greve, Sensors \& Actuators 17, 531 (1989).

[18] M.Y. Keating, G.A. Rechnitz, Anal. Chem. 57, 1998 (1984).

[19] J. Roncali, Chem. Rev. 92, 711 (1992).

[20] T.M. Swanger, M.J. Marsella, L. Bicknell, Qin Zhou, ACS Polym. Preprits 35, (1) 206 (1994); IUPAC Macro-Acron 94, Abstracts, p. 708.

[21] M.J. Marsella, T.M. Swanger, IUPAC Macro-Acron 94, Abstracts, p. 380; ACS Polymer Preprints 35, (1) 271 (1994).

[22] G.T. Hermans, A. Krishna Malia, P.K. Smith, Immobilized Affinity Ligand Techniques, Academic Press, New York 1992.

[23] T. Basińska, S. Słomkowski, M. Delmar, J. Bioact. Compt. Polym. 8, 205 (1993).

[24] B. Miksa, S. Slomkowski, Colloid. Polym. Sci., in press 1994.

[25] S. Słomkowski, D. Kowalczyk, M. Trznadel, M. Kryszewski, ACS Polym. Prep. 35, 409 (1994). 